\title{
Arthroscopic suprascapular neurectomy for the management of severe shoulder pain.
}

\begin{abstract}
Hypothesis: Arthroscopic suprascapular neurectomy is an effective option in the management of patients with severe shoulder pain. Materials and methods: We describe and evaluate a technique of suprascapular neurectomy, performed arthroscopically for the treatment of severe shoulder pain in 20 patients (17 with a rotator cuff arthropathy, two with glenohumeral arthritis and one with a rotator cuff deficient shoulder following an unsuccessful arthrodesis). Post-operative pain relief was measured using a new pain scoring system, which combined an assessment of the frequency and severity of pain experienced at night, at rest, with activities and any change in analgesic consumption. Results: At an average follow-up of 29 months, $75 \%$ of our patients reported good to excellent pain relief scores, $85 \%$ reported less night pain, $90 \%$ had less rest pain, $70 \%$ reported less pain on movement, whilst $75 \%$ reported less consumption of pain medication. There were no surgical complications. Discussion: Suprascapular neurectomy performed arthroscopically provides an additional surgical option in the management of pain in patients with cuff tear arthropathy and in other selected patients with no functioning rotator cuff. The pain scoring system introduced in this article provides a more comprehensive assessment of shoulder pain than existing pain scores. Conclusion: We conclude that arthroscopic suprascapular neurectomy introduces an additional effective option in the management of pain in patients with these pathologies. Level of evidence: Level 4; Retrospective case series, no control group.
\end{abstract}

Keyword: Arthroscopic suprascapular neurectomy; Pain scoring system; Rotator cuff arthropathy; Shoulder pain 